\title{
Cell programming: jumping back to pluripotency or rewiring fate
}

"The field has evolved from considering differentiation as irreversible to acceptance of the notion that cell fate is plastic and highly amenable to manipulation. This has profound implications for understanding disease biology and is expected to lead to fundamental breakthroughs in personalized and regenerative medicine."

During development cells with a broad potential differentiate into highly specialized cells with limited or no differentiation potential. Even though these differentiated cells share the same genotype, they have diverse cellular functions and possess distinct, yet stable, gene expression networks. Differentiation is often described in the context of epigenetic landscapes [1]. In this metaphor, cells are depicted as rolling down bifurcating valleys on an epigenetic hillside, thereby progressively acquiring diverse cell fates. Terminally differentiated cells reside at the bottom of the landscape in a stable state that is thought to be irreversible and unchangeable under homeostatic or physiological conditions.

Seemingly inconsistent with the stability of differentiated states are the results of experiments involving nuclear transfer, cell fusion and the introduction of specific transcription factors $[2,3]$. These experiments highlight the plasticity of cellular epigenomes and demonstrate that the states of differentiated cells can be reversed (e.g., reprogramming a nucleus to a more potent state) or directly converted from one specialized state to another (a process referred to as transdifferentiation).

The birth of Dolly, the first mammal generated from a reprogrammed adult differentiated cell and the first derivation of human embryonic stem cells, showed a path towards the generation of autologous pluripotent cells. Such cells could be used to model human disease, advance drug discovery and develop cell replacement therapies, which all require a renewable source of adult differentiated diseaserelevant cells [4-6]. A patient's easily accessible somatic cells could be isolated and injected into a donated egg, which could then be used to derive embryonic stem cells. These self-renewing pluripotent cells could be differentiated into disease-relevant cell types to establish novel model systems, or have any genetic defects repaired and then be transplanted back into the patient [7-9]. The ethical, technical and logistical concerns surrounding egg donation and the generation of human embryos for research, however, stalled the translation of this somatic cell transplantation-mediated reprogramming approach into humans.

There is a rich history to reprogramming and transdifferentiation research, starting in the 1950s with sets of experiments designed to determine if the genome were irreversibly modified through development and cell type specialization [3]. Unfortunately, the literature was contaminated with questionable reports of cell transdifferentiation and cell type plasticity. Most of these examples were later explained as cell fusion events, and many other examples have proven refractory to independent confirmation. These missteps served a useful purpose, however, by reminding investigators to remain vigilant and scrutinize all claims of cell fate reprogramming.

In addition to using a whole cell as a reprogramming medium, as for cell fusion or somatic cell nuclear transplantation, a long standing concept and approach has been to use defined cell type-specific factors to transdifferentiate one differentiated cell type into another. Elegant experiments reported in the 1980s by Davis et al. demonstrated that mouse embryonic fibroblasts could be programmed to acquire a myoblast-like cell identity by introducing a single transcription factor, $\mathrm{MyoD}$ [10]. Another example illustrating the potential for this principle was reported in 2004 when Xie et al. demonstrated that transcription factors C/EBPA and C/EBPB could program B cells into macrophages [11]. These reports demonstrated that specific transcription factors could
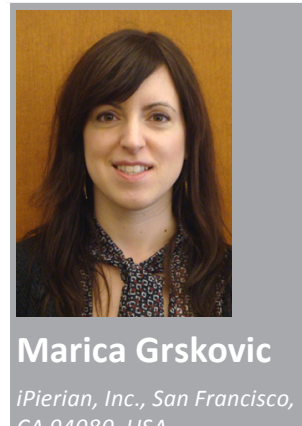
CA 94080, USA

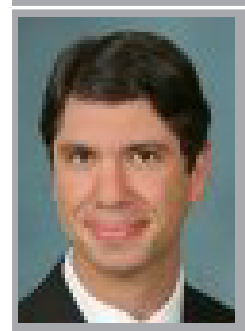

Ken Seidenman iPierian, Inc., San Francisco, CA 94080, USA

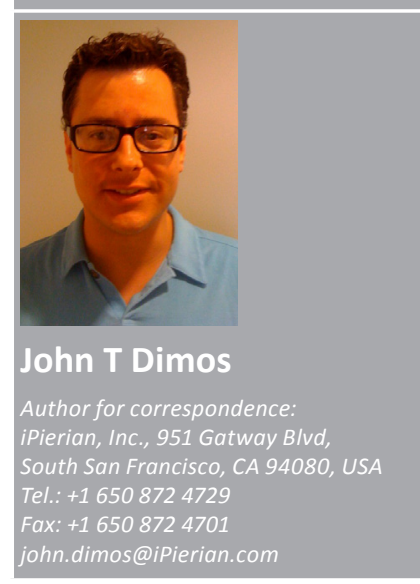


perturb stable gene expression networks and convert one specialized cell state to another, at least within the same embryonic germ layer. These reports, however, were taken as more a curiosity rather than breakthroughs with potential to revolutionize biomedicine.

Shinya Yamanaka's seminal announcement that the introduction of four transcriptional regulators could change the identity of mouse skin fibroblasts into cells closely resembling pluripotent embryonic stem cells energized the field in 2006 [12]. This breakthrough again opened the possibility of generating autologous pluripotent cells from patients for disease modeling, drug discovery and regenerative medicine, and reports of that development quickly followed $[13,14]$. Indeed, forced expression of three key transcription factors in readily accessible adult human skin fibroblasts leads to the acquisition of a pluripotent cell state from which, at least in principle, any other differentiated cell type can be generated [15].

\section{"Directed transdifferentiation offers the hope of cell therapy without the need for cell transplantation, as recently exemplified by the conversion of endocrine to excocrine cells in the pancreas of mice..."}

Capturing a semi-stable pluripotent stem cell intermediary is likely not required for transdifferentiation, as Vierbuchen et al. have reminded us recently [16]. While earlier studies clearly demonstrated that it was possible to convert one cell type into another by introducing a limited number of transcription factors, all examples were either cell type changes within the same germ layer lineage (e.g., mesodermal) or dedifferentiation into a pluripotent cell type. The recent study by Vierbuchen et al. achieved the remarkable feat of converting mouse fibroblasts into functionally validated neurons using as few as three genes, thus proving that it is indeed possible to convert cells originating from one germ layer (mesoderm) into cells of another (ectoderm) [16].

Vierbuchen et al. sought to identify a minimal set of factors that could transdifferentiate skin fibroblasts into neurons. They first prepared fibroblasts from knock-in mice whose genome harbored a fluorescent reporter controlled by the neuron-specific promoter Tau, then transduced these fibroblasts with pools of viruses expressing neural-specific genes. Approximately a month following gene transduction, occasional Tau$\mathrm{EGFP}^{+}$cells with typical neuronal morphologies appeared. Three key genes were found sufficient to rapidly convert approximately $2-8 \%$ of starting mouse fibroblasts into neurons [16].

Critically, the morphological changes observed in these cells were paralleled by functional changes in the passive and active membrane properties of the cells. Over a prolonged period, these induced neurons (iNeurons) showed increasing resting membrane potential, spontaneous action potentials and $\alpha$-amino-3-hydroxyl-5-methyl-4isoxazole-propionate $/ N$-methyl-D-aspartic acid currents, consistent with neuronal maturation. Furthermore, when co-cultured with astrocytes, iNeurons formed functional synapses with each other and with neonatal cortical neurons. While both glutamatergic and GABAergic neurons were detected in the iNeuron cultures, no dopaminergic, cholinergic or serotonergic neurons could be identified. This study illustrates that with as few as three genes, mouse fibroblasts can be transdifferentiated into functional neurons [16]. With the hope that this pioneering work will eventually translate to human cells, the potential biomedical applications of this technology are discussed below.

Perhaps the most promising potential application of this technology is the generation of patient-specific neurons for cell replacement therapy in neurodegenerative diseases. The use of patient-specific iNeurons would circumvent the need for fetal-derived neurons or embryonic stem cells, which, although promising, are fraught with ethical and political controversy. There are a number of fundamental issues that need to be addressed before direct transdifferentiation can be applied to cell therapy. A key question is to what extent it will be possible to generate CNS cell types other than glutamatergic and GABAergic neurons. This is an important consideration, as other cell types are among those primarily affected in many neurodegenerative diseases, for example dopaminergic neurons in Parkinson's disease, and cholinergic neurons and astrocytes in Alzheimer's disease and motor neuron diseases. The use of additional factors, altered culture conditions or both should allow for the generation of specific cell types. Of note, the screen conducted by Verbuchen et al. evaluated the efficiency of factor combinations based on the number of cells expressing a pan-neuronal marker irrespective of neuronal subtype. It is perhaps not unexpected that the most efficient combination of factors yields, almost exclusively, glutamatergic neurons, which are the most abundant neural cell type in the CNS. More subtypespecific neuronal reporter systems should enable 
combinatorial factor screens to yield other desired neuronal subtypes. It is exciting to speculate how, for example, glial cells in the spinal cord could be transdifferentiated into motor neurons within the context of a motor neuron degenerative disease patient. Directed transdifferentiation offers the hope of cell therapy without the need for cell transplantation, as recently exemplified by the conversion of endocrine to excocrine cells in the pancreas of mice $[17,18]$.

Patient-derived cells offer a promising system for drug discovery, as they provide a highly disease-relevant cellular and phenotypic context, and may predict the efficacy and toxicity of candidate therapeutic compounds in patients. For the majority of diseases it is clear that multiple cell types play an important role in pathogenesis. Therefore, the capacity of iPS cells to generate multiple cell types across lineages in a scalable and reproducible manner is an important advantage over transdifferentiation. Transdifferentiation technology, however, could allow for the rapid generation of a particular cell type, which could be useful for generating limited numbers of disease-relevant cells from a large panel of patients, for example when testing a late stage drug candidate for efficacy (an in vitro clinical trial) prior to testing in patients.

The field has evolved from considering differentiation as irreversible to acceptance of the notion that cell fate is plastic and highly amenable to manipulation. This has profound implications for understanding disease biology and is expected to lead to fundamental breakthroughs in personalized and regenerative medicine. While there is still much to learn about reprogramming and rewiring cell fates, it is already clear that these technologies will revolutionize biomedical research.

\section{Financial \& competing interests disclosure}

The authors are employees of iPierian, a company which holds financial stock in induced pluripotent stem cell technology. The authors have no other relevant affliations or financial involvement with any organization or entity with a financial interest in or financial conflict with the subject matter or materials discussed in the manuscript apart from those disclosed.

No writing assistance was utilized in the production of this manuscript.

\section{Bibliography}

1 Waddington CH: The Strategy of Genes. George Allen and Unwin, London, UK (1957).

2 Gurdon JB, Melton DA: Nuclear reprogramming in cells. Science 322(5909), 1811-1815 (2008).

3 Gurdon JB, Byrne JA: The first half-century of nuclear transplantation. Proc. Natl Acad. Sci. USA 100(14), 8048-8052 (2003).

4 Gurdon JB, Byrne JA, Simonsson S: Nuclear reprogramming and stem cell creation. Proc. Natl Acad. Sci. USA 100 (Suppl. 1), 11819-11822 (2003).

5 Eggan K: Using stem cells and reprogramming to understand disease. Regen. Med. 3(6), 799-801 (2008).

6 Eggan K: Dolly's legacy: human nuclear transplantation and better medicines for our children. Cloning Stem Cells 9(1), 21-25 (2007).

7 Daley GQ: Towards the generation of patient-specific pluripotent stem cells for combined gene and cell therapy of hematologic disorders. Hematology Am. Soc. Hematol. Educ. Program 17-22 (2007).

8 Daley GQ, Scadden DT: Prospects for stem cell-based therapy. Cell 132(4), 544-548 (2008).

9 Kazuki Y, Hiratsuka M, Takiguchi M et al.: Complete genetic correction of iPS cells from Duchenne muscular dystrophy. Mol. Ther. 18(2), 386-393 (2010).

10 Davis RL, Weintraub H, Lassar AB: Expression of a single transfected cDNA converts fibroblasts to myoblasts. Cell 51(6), 987-1000 (1987).

11 Xie H, Ye M, Feng R, Graf T: Stepwise reprogramming of $\mathrm{B}$ cells into macrophages. Cell 117(5), 663-676 (2004).

12 Takahashi K, Yamanaka S: Induction of pluripotent stem cells from mouse embryonic and adult fibroblast cultures by defined factors. Cell 126(4), 663-676 (2006).

13 Dimos JT, Rodolfa KT, Niakan KK et al:: Induced pluripotent stem cells generated from patients with ALS can be differentiated into motor neurons. Science 321(5893), 1218-1221 (2008).

14 Park IH, Arora N, Huo H et al.: Disease-specific induced pluripotent stem cells. Cell 134(5), 877-886 (2008).

15 Nakagawa M, Koyanagi M, Tanabe K et al.: Generation of induced pluripotent stem cells without Myc from mouse and human fibroblasts. Nat. Biotechnol 26(1), 101-106 (2008).

16 Vierbuchen T, Ostermeier A, Pang ZP, Kokubu Y, Sudhof TC, Wernig M: Direct conversion of fibroblasts to functional neurons by defined factors. Nature 463(7284), 1035-1041 (2010).

17 Zhou Q, Melton DA: Extreme makeover: converting one cell into another. Cell Stem Cell 3(4), 382-388 (2008).

18 Zhou Q, Brown J, Kanarek A, Rajagopal J, Melton DA: In vivo reprogramming of adult pancreatic exocrine cells to $\beta$-cells. Nature 455(7213), 627-632 (2008). 